\title{
A Study of Operator and Parameter Choices in Non-Revisiting Genetic Algorithm
}

\author{
Shiu Yin Yuen and Chi Kin Chow
}

\begin{abstract}
We study empirically the effects of operator and parameter choices on the performance of the non-revisiting genetic algorithm (NrGA). For a suite of 14 benchmark functions that include both uni-modal and multi-modal functions, it is found that NrGA is insensitive to the axis resolution of the problem, which is a good feature. From the empirical experiments, for operators, it is found that crossover is an essential operator for NrGA, and the best crossover operator is uniform crossover, while the best selection operator is elitist selection. For parameters, a small population, with a population size strictly larger than 1 , should be used; the performance is monotonically increasing with crossover rate and the optimal crossover rate is 0.5 . The results of this paper provide empirical guidelines for operator designs and parameter settings of NrGA.
\end{abstract}

\section{INTRODUCTION}

G uidleines on operator choices and parameter values are valuable information for industry practitioners and researchers who wish to use an algorithm to solve their research problems. In this paper, we empirically study the effects of applying different operators and parameter values to the non-revisiting genetic algorithm (NrGA) [1, 2].

The original motivation of NrGA aims to answer a fundamental question: can a stochastic search algorithm - in this case a GA - be designed such that the search will never revisit a location that has been visited before? For deterministic search problems, the answer is simple because one can always search in a particular order. For stochastic search, in general, one needs to remember every visited search positions. Is that worthwhile or feasible?

The answer is definitely yes for applications involving expensive or time consuming fitness evaluations, or both. Many day to day engineering applications fall into this category. For such applications, a fitness evaluation may take from 1 second to 1 day. Some examples are antenna design [3], heating, ventilating and air conditioning (HVAC) engineering [4] and three dimensional object registration in computer vision [5]. In such application, it is only natural to store all fitness evaluation results. Since the total number of evaluations is limited, the memory required is acceptable.

Shiu Yin Yuen is with the Department of Electronic Engineering, City University of Hong Kong, Hong Kong SAR, China (E-mail: kelviny.ee@cityu.edu.hk).

Chi Kin Chow is with the Department of Electronic Engineering, City University of Hong Kong, Hong Kong SAR, China (E-mail: chowchi@cityu.edu.hk).

The work described in this paper was supported by CityU Research Grant (7002304).
This is especially so for future evolutionary algorithms as memory available is increasing rapidly due to Moore's law.

In addition to storing all visited solutions, $\mathrm{NrGA}$ aims at storing and organizing the entire search history in a way to make intelligent search decisions actively. A first demonstration, as used in NrGA, is to use a binary space partitioning (BSP) tree in computational geometry [ 6] to organize the visited solutions. This gives naturally an adaptive mutation operator that has no parameter. Consequently, the NrGA has very few parameters. The following are its parameters: (1) axis resolution parameter $d$; this is the number of discrete levels for each gene; (2) $\mu$ and $\lambda$ for the $(\mu+\lambda)$ selection; and (3) crossover rate $r_{x}$. $d$ is application dependent. In [2], $\mu=100, \lambda=200, r_{x}=0.5$.

In [2], the operator choices are: (1) elitist $(\mu+\lambda)$ selection and (2) uniform crossover.

An optional background mutation operator of $1 / D$ type may also be added, where $D$ is the problem dimension. It is turned on in [1] and is turned off in [2] (to better illustrate the performance gain due to the parameter-less adaptive mutation.)

The NrGA design is illustrated in Figure 1. As seen, it can be appreciated that it is just a simple GA coupled with an adaptive mutation module.

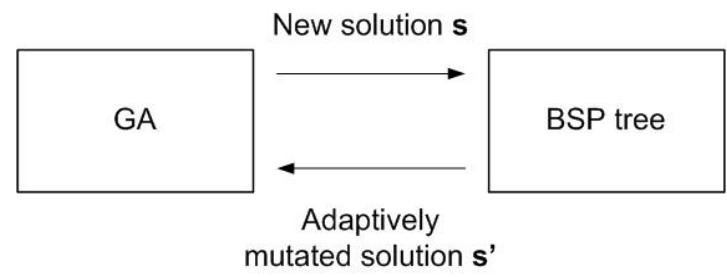

Figure 1. Communication between GA and the BSP tree

NrGA was compared with (1) real coded GA (RC-GA); (2) Covariance Matrix Adaptation Evolution Strategy (CMA-ES) - a state of the art adaptive mutation method; (3) canonical GA (CGA); (4) GA with a simple diversity mechanism (Div-GA) and (5) three forms of enhanced particle swarm optimization (DPSO, SEPSO and PSOMS). The test was performed on 19 well known benchmark functions in a total 64 test cases. It is observed that NrGA outperforms the other test algorithms: NrGA ranks first (or joint first) in 39 and is second in 13 out of the 64 cases. For full details of NrGA, please refer to [2].

Particularly worthy of mention is that NrGA outperforms CMA-ES, which is the choice method recommended by experts for adaptive mutation [7]. Note that in CMA-ES, the 
parameter settings have been fine tuned by hand.

This motivates us to study why NrGA works. In particular, we wish to answer the question: what is the influence of operator and parameter choices and settings in NrGA designs? Is there a recommended design for NrGA? In this paper, we study the effects of operator choices and parameter settings in NrGA: We did control experiments on 1) population size $\mu=\lambda$. ( $\lambda$ is set equal to $\mu$.) 2) axis resolution $d, 3$ ) crossover rate $\left.r_{x}, 4\right)$ crossover operator and 5) selection scheme. We shall draw some conclusions based on the results of these empirical experiments.

The rest of this paper is organized as follows: Section II explains the simulation setup. Section III reports the results on varying population size. Section IV reports the results of changing the axis resolution. Section $V$ reports the effect of varying the crossover rate. Section VI reports the effects of different crossover operators. Section VII reports on using different selection schemes. Section VIII gives the conclusion.

\section{Simulation SetuP}

In this paper, we qualify the performance of NrGA by its best fitness. The performance of NrGA for a particular setting at a particular objective function is concluded by the averaged best fitness of 100 independent runs.

\section{A. Test functions}

A real valued function set $\mathbf{F}=\left\{f_{1}(\mathbf{x}), f_{2}(\mathbf{x}), \ldots, f_{14}(\mathbf{x})\right\}$ consisting of 14 well known benchmark functions are employed. The test functions, which can be found in [8], are as follows:

1. Sphere function

2. Schwefel's problem 2.22

3. Schwefel's problem 1.2

4. Schwefel's problem 2.21

5. Generalized Rosenbrock function

6. Quartic function

7. Generalized Rastrigin function

8. Generalized Griewank function

9. Generalized Schwefel's problem 2.26

10. Ackley function

11. Shekel's Foxholes

12. Six-Hump Camel-Back function

13. Branin function

14. Goldstein-Price function

The mathematical forms, the search space and the optima of these functions are given Table 5.

The first six functions are uni-modal functions; the remaining eight are multi-modal functions designed with a considerable amount of local minima. The dimensions of the first ten functions are adjustable while the dimensions of $f_{11}$ $f_{14}$ are fixed at two. All functions with the exceptions of $f_{9}, f_{11}$, $f_{12}$ and $f_{13}$, have the global minimum at the origin or very close to the origin. Simulations are carried out to find the global minimum of each function.

\section{B. Basic setting of $\mathrm{NrGA}$}

In this paper, we examine the sensitivities of six operators /parameters of NrGA including: 1) population size $\mu, 2)$ axis resolution $d, 3)$ crossover rate $\left.r_{x}, 4\right)$ crossover operator $C($.$) ,$ and 5) selection scheme $S($.$) . In each of the sensitivity$ analyses, only one of the operators /parameters is varied and we observe the influence to the averaged best fitness value. In all the experiments, NrGA has the following settings throughout this paper unless specified otherwise:

- Population size $\mu=100$

- Numbers of generations $N_{g}$ : 400 for the first ten test functions and 40 for the last four test functions.

- Axis resolution $d$ : 100 for the first ten functions and 2000 for the last four test functions.

- Number of dimensions $D: 30$ for the first ten test functions and 2 for the last four test functions.

- Crossover operator $C()=$. uniform crossover

- Crossover rate $r_{x}=0.5$

- Selection scheme $S($.$) = elitism selection$

For example, in section IV, the parameters except population size $\mu$ is assigned as: $d=100, N_{g}=400$ or $40, C($.) is uniform crossover, $r_{x}=0.5, \mathrm{~S}()=$. elitism selection and $D=$ 30. Similarly, in section $\mathrm{V}$, the parameters except axis resolution $d$ are assigned as: $\mu=100, N_{g}=400$ or $40, C($.$) is$ uniform crossover, $r_{x}=0.5, S()=$. elitism selection and $D=$ 30 .

\section{Population SizE}

In this experiment, we examine the performance of NrGA for different population sizes. For test functions $f_{1}-f_{10}$, the population size is varied from 2 to 20,000. Table 1 lists the combinations of population size $\mu$ against number of generation $N_{g}$. The total number of evaluations for $f_{1}-f_{10}$ is kept constant at 40,000.

For test functions $f_{11}-f_{14}$, the population size is varied from 2 to 2,000. Table 2 lists the combinations of $\mu$ and $N_{g}$. The total number of evaluations for $f_{1}-f_{10}$ is kept constant at 2,000 .

Figure 2 shows the averaged best fitness value against population size for $f_{1}-f_{14}$. The general trend is that the averaged best fitness value increases with population size. So the population size should be kept small. For $f_{1}-f_{10}$, the optimal population size is 2 ; for $f_{11}-f_{14}$, the optimal population size is small. Note that the population size cannot be 1 , otherwise the crossover operator degenerates and there is a drastic drop in performance (see section $\mathrm{V}$ ).

TABLE 1. THE COMBINATIONS OF POPULATION SIZE AND NUMBER OF GENERATIONS FOR $f_{l}-f_{10}$ IN EXPERIMENT 1 .

\begin{tabular}{ll|ll|ll}
$\mu$ & $N_{g}$ & $\mu$ & $N_{g}$ & $\mu$ & $N_{g}$ \\
\hline
\end{tabular}




\begin{tabular}{cc|cc|cc}
\hline 2 & 20,000 & 80 & 500 & 1,250 & 32 \\
4 & 10,000 & 100 & 400 & 1,600 & 25 \\
5 & 8,000 & 125 & 320 & 2,000 & 20 \\
8 & 5,000 & 160 & 250 & 2,500 & 16 \\
10 & 4,000 & 200 & 200 & 4,000 & 10 \\
16 & 2,500 & 250 & 160 & 5,000 & 8 \\
20 & 2,000 & 320 & 125 & 8,000 & 5 \\
25 & 1,600 & 400 & 100 & 10,000 & 4 \\
32 & 1,250 & 500 & 80 & 20,000 & 2 \\
40 & 1,000 & 625 & 64 & & \\
50 & 800 & 800 & 50 & & \\
64 & 625 & 1,000 & 40 & & \\
\hline
\end{tabular}

TABLE 2.THE COMBINATIONS OF POPULATION SIZE AND NUMBER OF GENERATIONS FOR $f_{11}-f_{14}$ IN EXPERIMENT 1

\begin{tabular}{cc|cc|cc}
\hline$\mu$ & $N_{g}$ & $\mu$ & $N_{g}$ & $\mu$ & $N_{g}$ \\
\hline 2 & 2,000 & 32 & 125 & 250 & 16 \\
4 & 1,000 & 40 & 100 & 400 & 10 \\
5 & 800 & 50 & 80 & 500 & 8 \\
8 & 500 & 80 & 50 & 800 & 5 \\
10 & 400 & 100 & 40 & 1,000 & 4 \\
16 & 250 & 125 & 32 & 2,000 & 2 \\
20 & 200 & 160 & 25 & & \\
25 & 160 & 200 & 20 & & \\
\hline
\end{tabular}

\section{Axis Resolution}

Axis resolution $d$ directly affects the precision of the optimum found by $\mathrm{NrGA}$; the precision is raised by increasing the resolution. According to the adaptive mutation scheme of $\mathrm{NrGA}$, the mutation step size depends on the size of the unvisited subspace rather than the resolution. Nonetheless, if the resolution is low, the chance of revisit and hence node pruning occurs more frequently. These will benefit NrGA in two ways: firstly, node pruning will simplify the BSP tree and the time for node search will be shorter. Also, NrGA can escape from a local optimum when the unvisited subspace of the local optimum is fully evaluated.

However, as the resolution increases, node pruning rarely occurs. As the mutation step size is independent of the resolution, the performance of $\mathrm{NrGA}$ should be invariant to the resolution when it is sufficiently high. This predicted performance is shown in Figure 3. When $d \leq 2^{13}=8192$ (i.e. the usual range settings for GA), the performance is unchanged when $d$ is varied. Interestingly, as the resolution increases from $2^{15}$ to $2^{20}$, i.e. the resolution is close to being continuous, the performance is also unchanged.

\section{Crossover Rate}

In this experiment, we examine the performance of NrGA for crossover rate $r_{x}$ at $0.0,0.05, \ldots, 0.5$. TABLE 6 and TABLE 7 show the averaged best fitness values against crossover rate for $f_{1}-f_{14}$. Seen from the figures, the averaged best fitness monotonically decreases with increases in crossover rate. The results suggest that the optimal crossover rate (using uniform crossover) is 0.5 .

Comparing the first two rows of the tables, it can be observed that there is a drastic performance improvement when $r_{x}$ increases from 0 to 0.05 . This suggests that the crossover operator is an essential operator in NrGA.

\section{CROSSOVER OPERATOR}

In this experiment, we examine the performance of $\mathrm{NrGA}$ for four crossover operators: 1) Uniform crossover, 2) 1-point crossover, 3) 2-point crossover and 4) Arithmetic crossover. For uniform crossover, the crossover rate is 0.5 . For the 1-point and 2-point crossover operators, the crossover boundaries are randomly selected. For arithmetic crossover, the crossover weight is randomly selected between $[0,1]$. Since the 2-point crossover operator involves two crossover boundaries, an individual must have at least three genes. So the dimension of the corresponding objective function should be at least three. Thus only the first ten test functions $f_{1}-f_{10}$ are employed in this experiment.

Table 3 lists the averaged best fitness for the four crossover operators. The values inside the brackets indicate the standard deviations of the fitness values. The crossover operator with the smallest fitness value for each test function is in bold. Note that there is a considerable performance difference amongst the operators. This suggests that the performance of $\mathrm{NrGA}$ is sensitive to the crossover operator choice.

Seen from the table, the uniform crossover ranks first (or joint first) in 8 out of 10 test functions. Thus, it is suggested to employ the uniform crossover operator in NrGA when there is no knowledge about the selection of crossover operator. To illustrate the significance of the recommendation of uniform crossover, the confidence interval (in term of \%) for t-test comparing the averaged best fitness values of the uniform crossover with the other three operators are listed. The confidence intervals of the uniform crossover compared to another operator is indicated by "-" if the averaged best fitness values of both operators are equal.

The significance of the rank of the uniform crossover is summarized as follows: We are $99.95 \%$ confident that the uniform crossover is the best among the four operators in 4 test functions. For the remaining four functions that the uniform crossover ranks or jointly ranks first, one out of them is with $99.75 \%$ confidence interval, and the remaining three are with $95 \%$ confidence interval. From the above, we conclude that the recommendation of crossover operator is statistically significant.

TABLE 3. AVERAGED BEST FITNESS OF THE TEST FUNCTIONS FOR DIFFERENT CROSSOVER-OPERATORS

\begin{tabular}{|c|c|c|c|c|}
\hline & Uniform & 1-point & 2-point & Arithmetic \\
\hline \multirow{3}{*}{$f_{1}$} & 0.000 & 0.0108 & 0.000 & 8.3768 \\
\hline & $(0.000)$ & $(0.0601)$ & $(0.000)$ & $(6.667)$ \\
\hline & - & $95 \%$ & - & $99.95 \%$ \\
\hline \multirow{3}{*}{$f_{2}$} & 0.000 & 0.516 & 0.124 & 15.054 \\
\hline & $(0.000)$ & (1.2179) & $(0.665)$ & $(3.176)$ \\
\hline & - & $99.95 \%$ & $95 \%$ & $99.95 \%$ \\
\hline \multirow{3}{*}{$f_{3}$} & 2554.04 & 6222.96 & 5325.88 & 8156.6 \\
\hline & $(1353.7626)$ & $(2526.581)$ & (1956.1209) & $(3155.758)$ \\
\hline & - & $99.95 \%$ & $99.95 \%$ & $99.95 \%$ \\
\hline \multirow{3}{*}{$f_{4}$} & 35.28 & 64.16 & 61.58 & 19.1 \\
\hline & $(5.3853)$ & $(5.3865)$ & $(6.4516)$ & $(2.7622)$ \\
\hline & - & $99.95 \%$ & $99.95 \%$ & $99.95 \%$ \\
\hline \multirow{3}{*}{$f_{5}$} & 463.1292 & 4696.4448 & 1351.2924 & 194773.68 \\
\hline & (653.95) & (11442.63) & (2921.70) & (119623.79) \\
\hline & - & $99.95 \%$ & $99.75 \%$ & $99.95 \%$ \\
\hline$f_{\kappa}$ & 8.6445 & 16.4004 & 13.394 & 8.4447 \\
\hline
\end{tabular}




\begin{tabular}{ccccc} 
& $(0.5138)$ & $(2.5817)$ & $(2.0214)$ & $\mathbf{( 0 . 4 9 0 7 )}$ \\
& - & $99.95 \%$ & $99.95 \%$ & $\mathbf{9 9 . 5 0 \%}$ \\
\hline \multirow{2}{*}{$f_{7}$} & $\mathbf{1 6 . 4 1 7 8}$ & 69.9228 & 56.849 & 64.6156 \\
& $\mathbf{( 5 . 7 4 3 8 )}$ & $(16.2481)$ & $(14.8125)$ & $(9.0986)$ \\
& - & $99.95 \%$ & $99.95 \%$ & $99.95 \%$ \\
\hline \multirow{2}{*}{$f_{8}$} & $\mathbf{0 . 0 0 0}$ & 0.0569 & 0.003 & 16.6708 \\
& $\mathbf{( 0 . 0 0 0 )}$ & $(0.1365)$ & $(0.0173)$ & $(5.8675)$ \\
& - & $99.95 \%$ & $95 \%$ & $99.95 \%$ \\
\hline \multirow{2}{*}{$f_{9}$} & $\mathbf{- 1 3 0 4 7 . 6 3 2 5}$ & -10752.4713 & -11175.6634 & -6294.315 \\
& $\mathbf{( 2 2 7 . 4 7 3 5 )}$ & $(616.7379)$ & $(548.9175)$ & $(751.7627)$ \\
& - & $99.95 \%$ & $99.95 \%$ & $99.95 \%$ \\
\hline \multirow{2}{*}{$f_{10}$} & $\mathbf{0 . 0 0 0}$ & 11.3348 & 5.1462 & 9.1697 \\
& $\mathbf{( 0 . 0 0 0 )}$ & $(4.5851)$ & $(4.7577)$ & $(1.0871)$ \\
\hline \multicolumn{6}{c}{} & - & $99.95 \%$ & $99.95 \%$ & $99.95 \%$ \\
\hline
\end{tabular}

\section{SELECTION SCHEME}

In this experiment, we examine the performance of $\mathrm{NrGA}$ for three selection schemes:

1. Elitist selection (elitism) - Suppose $\mathbf{X}=\left\{\mathbf{x}_{\mathbf{i}}\right\}_{i=1,2, \ldots, n}$ is the parent pool and $\mathbf{O}=\left\{\mathbf{o}_{\mathbf{i}}\right\}_{i=1,2, \ldots, n}$ is the offspring pool. Elitist selection chooses the $n$ individual with the smallest fitness from the mixture of the pools $\mathbf{X}$ and $\mathbf{O}$ to form the new population.

2. Rank-based proportional selection (proportional) Suppose $\mathbf{X}=\left\{\mathbf{x}_{\mathbf{i}}\right\}_{i=1,2, \ldots, n}$ is the parent pool and $\mathbf{O}=$ $\left\{\mathbf{0}_{\mathbf{i}}\right\}_{i=1,2, \ldots, n}$ is the offspring pool, $\mathbf{M}=\left\{\mathbf{m}_{\mathbf{i}}\right\}_{i=1,2, \ldots, 2 n}=\{\mathbf{X}$, $\mathbf{O}\}$ is the mixture of the pools $\mathbf{X}$ and $\mathbf{O}$, the probability that $\mathbf{m}_{\mathbf{j}} \in \mathbf{M}$ is chosen as the member of the new population is:

$$
\left(2 n-r_{j}+1\right) \times\left(\sum_{i=1}^{2 n} i\right)^{-1}
$$

where $r_{j}$ is the rank of $\mathbf{m}_{\mathbf{j}}$ in $\mathbf{M}$.

3. Tournament selection (tournament) - Suppose $\mathbf{X}=$ $\left\{\mathbf{x}_{\mathbf{i}}\right\}_{i=1,2, \ldots, n}$ is the parent pool, every individual $\mathbf{o}_{\mathbf{k}}$ in the offspring pool $\mathbf{O}=\left\{\mathbf{o}_{\mathbf{i}}\right\}_{i=1,2, \ldots, n}$ is generated as follows: Given a tournament size $t$, we first randomly choose $t$ individuals from $\mathrm{X}$ and form the sub-pool $S=\left\{\mathbf{s}_{\mathbf{j}}\right\}_{j=1,2, \ldots, t}$ where $\mathbf{s}_{\mathbf{j}} \in \mathbf{X}$ for all $j$. The probability that $\mathbf{o}_{\mathbf{k}}$ is assigned as $\mathbf{s}_{\mathbf{j}}$ is:

$$
g_{k} \times\left(\sum_{j=1}^{t} g_{j}\right)^{-1}
$$

where $g_{k}=f\left(\mathbf{s}_{\mathbf{j}}\right)^{-1}$ and $f($.$) is the objective function. In this$ experiment, the tournament size is chosen to be 10 .

Table 4 lists the averaged best fitness for the three selection schemes. The values inside the brackets indicate the standard deviations of the fitness values. The selection scheme with the smallest fitness value for each test function is in bold. Seen from the table, the elitist selection ranks first for eleven test functions; the rank-based proportional selection performs best for the other three test functions.

Thus it is suggested to employ the elitism selection in NrGA. To illustrate the significance of the recommendation of this selection scheme, the confidence interval (in term of $\%$ ) for t-test comparing the averaged best fitness values of the elitism selection with the other selection schemes are listed.

The significance of the rank of the elitism selection is summarized as follows: We are $99.95 \%$ confident that the uniform crossover is the best among the four operators in seven test functions. For the remaining four functions that the elitism selection ranks first, one out of them is with $97.5 \%$ confidence interval, and the remaining three test functions are with $80 \%$ confidence interval. From the above, we conclude that the recommendation of selection scheme is statistically significant.

\begin{tabular}{|c|c|c|c|}
\hline & Elitism & Proportional & Tournament \\
\hline \multirow{3}{*}{$f_{l}$} & 0.000 & 0.0092 & 0.028 \\
\hline & $(0.000)$ & $(0.0168)$ & $(0.0421)$ \\
\hline & - & $99.95 \%$ & $99.95 \%$ \\
\hline \multirow{3}{*}{$f_{2}$} & 0.000 & 0.024 & 86.66 \\
\hline & $(0.000)$ & $(0.065)$ & (13.9124) \\
\hline & - & $99.95 \%$ & $99.95 \%$ \\
\hline \multirow{3}{*}{$f_{3}$} & 2431.76 & 6184.16 & 33120.76 \\
\hline & $(1090.6183)$ & $(2268.1166)$ & (8424.3433) \\
\hline & - & $99.95 \%$ & $99.95 \%$ \\
\hline \multirow{3}{*}{$f_{4}$} & 35.84 & 30.64 & 44.1 \\
\hline & $(5.9778)$ & (5.2603) & (7.8198) \\
\hline & - & $99.95 \%$ & $99.95 \%$ \\
\hline \multirow{3}{*}{$f_{5}$} & 513.99 & 725.7024 & 2829.114 \\
\hline & (713.6914) & $(777.2359)$ & $(4342.3787)$ \\
\hline & - & $97.5 \%$ & $99.95 \%$ \\
\hline \multirow{3}{*}{$f_{6}$} & 8.7656 & 8.5717 & 10.5206 \\
\hline & $(0.4943)$ & $(0.4579)$ & $(0.969)$ \\
\hline & - & $99.5 \%$ & $99.95 \%$ \\
\hline \multirow{3}{*}{$f_{7}$} & $\mathbf{1 7 . 5 2 2 4}$ & 28.9291 & 56.4179 \\
\hline & (4.9273) & $(7.8545)$ & (12.0177) \\
\hline & - & $99.95 \%$ & $99.95 \%$ \\
\hline \multirow{3}{*}{$f_{8}$} & 0.000 & 0.0518 & 0.1247 \\
\hline & $(0.000)$ & $(0.0634)$ & $(0.0932)$ \\
\hline & - & $99.95 \%$ & $99.95 \%$ \\
\hline \multirow{3}{*}{$f_{9}$} & -13034.4643 & -12623.4954 & -11432.4903 \\
\hline & (219.4799) & $(337.9245)$ & (503.3919) \\
\hline & - & $99.95 \%$ & $99.95 \%$ \\
\hline \multirow{3}{*}{$f_{10}$} & 0.000 & 0.0849 & 5.6249 \\
\hline & $(0.000)$ & $(0.2105)$ & $(4.6608)$ \\
\hline & - & $99.95 \%$ & $99.95 \%$ \\
\hline \multirow{3}{*}{$f_{11}$} & 2.3324 & 2.7103 & 3.0519 \\
\hline & (2.0394) & $(2.9352)$ & $(2.4955)$ \\
\hline & - & $80 \%$ & $97.5 \%$ \\
\hline \multirow{3}{*}{$f_{12}$} & -1.0277 & -1.0221 & -0.859 \\
\hline & $(0.0245)$ & $(0.057)$ & $(0.2463)$ \\
\hline & - & $80 \%$ & $99.95 \%$ \\
\hline \multirow{3}{*}{$f_{13}$} & 0.406 & 0.4143 & 0.7462 \\
\hline & $(0.0568)$ & $(0.0653)$ & $(0.6431)$ \\
\hline & - & $80 \%$ & $99.95 \%$ \\
\hline \multirow{3}{*}{$f_{14}$} & 3.871 & 3.7481 & 10.2517 \\
\hline & (3.9503) & (3.6385) & $(8.5555)$ \\
\hline & - & $<50 \%$ & $99.95 \%$ \\
\hline
\end{tabular}

TABLE 4. AVERAGED BEST FITNESS OF THE TEST FUNCTIONS FOR DIFFERENT

\section{CONCLUSION}

Non-revisiting genetic algorithm ( $\mathrm{NrGA})$ is a promising new evolutionary algorithm. It has superior performance compared with some well known evolutionary algorithms. Its merits are (1) simple design, with few parameters; (2) the use of the entire search history; (3) the ability to make intelligent decisions from all past knowledge gained. It features a 
powerful parameter-less adaptive mutation operator, which is parameter free but performs impressively well. This motivates us to investigate why the idea works.

In this paper, we report empirical investigations on the influence of operator and parameter choices on $\mathrm{NrGA}$. It is found that (1) a small population, roughly from 2 to 200 , gives the best performance; (2) the performance is stable and constant when the axis resolution parameter varies (a) within the ranges normally used in GA applications and (b) when the resolution parameter is close to continuous [9]; (3) crossover is an essential operator and should be used; (4) the best crossover operator is the uniform crossover; (5) the best crossover rate is 0.5 , the maximum rate possible; and (6) the best selection operator is the elitist selection operator.

The results of this paper contributes to the evolutionary computation community in three ways: (1) it shows that NrGA is insensitive to axis resolution, which makes it attractive to apply to diverse practical application; (2) it gives a set of empirical guidelines on operator and parameter choices of NrGA, which are useful information for industry practitioners interested in applying the NrGA to their engineering applications; and lastly, (3) it gives some data to theorists who wish to investigate a rigorous theoretical model of NrGA.

Instead of fixed operators and parameters, new schemes for operator and parameter control [7] for $\mathrm{NrGA}$ can be investigated in future, not withstanding that operator and parameter control is a very hard problem in general. It is interesting to note in passing that the parameter-less adaptive mutation in NrGA constitutes a form of parameter control that adaptively discovers its control mutation operators and mutation parameters from the search process itself.

\section{REFERENCES}

[ 1 ] S. Y. Yuen and C. K. Chow, "A Non-revisiting Genetic Algorithm", in Proc. IEEE CEC, pp. 4583 - 4590, 2007.

[2 2 S.Y. Yuen and C.K. Chow, "A Genetic algorithm that adaptively mutates and never revisits," IEEE Transactions on Evolutionary Computation, to be published.

[ 3 ] Q. X. Chu, K. F. Chan and C. H. Chan, "Parallel FDTD analysis of active integrated antenna array," Microwave and Optical Technology Letters, vol. 34, pp. 317-319, 2002.

[4] K. F. Fong, V. I. Hanby and T. T. Chow, "HVAC system optimization for energy management by evolutionary programming," Energy and Buildings, vol. 38, pp. 220-231, 2006.

[ 5 ] C. K. Chow, H. T. Tsui and T. Lee, "Surface registration using a dynamic genetic algorithm," Pattern Recognition, vol. 37, no. 1, pp. 105-117, 2004.

[6] M. de Berg, M. van Kreveld, M. Overmars and O. Schwarzkopf, Computational geometry, algorithms and applications, $2^{\text {nd }}$ Ed., 2000.

[7] F.G. Lobo, C.F. Lima and Z. Michalewicz (Eds.), Parameter setting in evolutionary algorithms, Springer, 2007.

[ 8 ] X. Yao, Y. Liu, and G. M. Lin, "Evolutionary programming made faster," IEEE Trans. Evolutionary Computation, vol. 3, no. 2, pp. 82-102, 1999.

[ 9 ] S.Y. Yuen and C.K. Chow, "Continuous non-revisiting genetic algorithm," in Proc. IEEE CEC, 2009.

TABLE 5 DETAILS OF THE FOURTEEN TEST FUCNTIONS.

\begin{tabular}{|c|c|c|c|}
\hline test function & mathematical form & range & optimum \\
\hline 1. Sphere function & $f_{1}(\mathbf{x})=\sum_{i=1}^{D} x_{i}^{2}$ & {$[-100,100]^{\mathrm{D}}$} & {$[0,0, \ldots, 0]$} \\
\hline 2. Schwefel's problem 2.22 & $f_{2}(\mathbf{x})=\sum_{i=1}^{D}\left|x_{i}\right|+\prod_{i=1}^{D}\left|x_{i}\right|$ & {$[-100,100]^{\mathrm{D}}$} & {$[0,0, \ldots, 0]$} \\
\hline 3. Schwefel's problem 1.2 & $f_{3}(\mathbf{x})=\sum_{i=1}^{D}\left(\sum_{j=1}^{i} x_{j}\right)$ & {$[-100,100]^{\mathrm{D}}$} & {$[0,0, \ldots, 0]$} \\
\hline 4. Schwefel's problem 2.21 & $f_{4}(\mathbf{x})=\max _{i \in[1, D]}\left|x_{i}\right|$ & {$[-100,100]^{\mathrm{D}}$} & {$[0,0, \ldots, 0]$} \\
\hline 5. Generalized Rosenbrock function & $f_{5}(\mathbf{x})=\sum_{i=1}^{D-1}\left[100\left(x_{i+1}-x_{i}^{2}\right)^{2}+\left(x_{i}-1\right)^{2}\right]$ & {$[-29,31]^{\mathrm{D}}$} & {$[1,1, \ldots, 1]$} \\
\hline 6. Quartic function & $\begin{array}{l}f_{6}(\mathbf{x})=\sum_{i=1}^{D} i x^{4}+\operatorname{random}[0,1] \\
\text { Note: This is a noisy fitness function. There is a } \\
\text { random measurement noise in each fitness evaluation. }\end{array}$ & {$[-1.28,1.25]^{\mathrm{D}}$} & {$[0,0, \ldots, 0]$} \\
\hline 7. Generalized Rastrigin function & $f_{7}(\mathbf{x})=\sum_{i=1}^{D}\left[x_{i}^{2}-10 \cos \left(2 \pi x_{i}\right)+10\right]$ & {$[-5.12,5.12]^{\mathrm{D}}$} & {$[0,0, \ldots, 0]$} \\
\hline 8. Generalized Griewank function & $f_{8}(\mathbf{x})=\frac{1}{4000} \sum_{i=1}^{D} x_{i}^{2}-\prod_{i=1}^{D} \cos \frac{x_{i}}{\sqrt{i}}+1$ & {$[-600,600]^{\mathrm{D}}$} & {$[0,0, \ldots, 0]$} \\
\hline 9. Generalized Schwefel's problem 2.26 & $f_{9}(\mathbf{x})=-\sum_{i=1}^{D} x_{i} \sin \sqrt{\left|x_{i}\right|}$ & {$[-500,500]^{\mathrm{D}}$} & $\begin{array}{c}{[420.9687, \ldots,} \\
420.9687]\end{array}$ \\
\hline 10. Ackley function & $\begin{aligned} f_{10}(\mathbf{x})= & -20 \exp \left(-0.2 \sqrt{\frac{1}{D} \sum_{i=1}^{D} x_{i}^{2}}\right)- \\
& \exp \left(\frac{1}{D} \sum_{i=1}^{D} \cos 2 \pi x_{i}\right)+20+e\end{aligned}$ & {$[-32,32]^{\mathrm{D}}$} & {$[0,0, \ldots, 0]$} \\
\hline
\end{tabular}


11. Shekel's Foxholes function

12. Six-Hump Camel-Back function

$$
f_{11}(\mathbf{x})=\left[\frac{1}{500}+\sum_{j=1}^{25} \frac{1}{j+\sum_{i=1}^{2}\left(x_{i}-a_{i, j}\right)^{6}}\right]^{-1} \text { where }
$$

$\left\{a_{i, j}\right\}=\left[\begin{array}{cccccccccc}-32 & -16 & 0 & 16 & 32 & -32 & \cdots & 0 & 16 & 32 \\ -32 & -32 & -32 & -32 & -32 & -16 & \cdots & 32 & 32 & 32\end{array}\right]$

$$
f_{12}(\mathbf{x})=4 x_{1}^{2}-2.1 x_{1}^{4}+\frac{1}{3} x_{1}^{6}+x_{1} x_{2}-4 x_{2}^{2}+4 x_{2}^{4}
$$

$[-4.91017$, $5.0893] \times$

$[-5.7126$,

4.2874]

$[0.08983,-0.7126]$ and $[-0.08983$, $0.7126]$

13. Branin function

$$
f_{13}(\mathbf{x})=\left(x_{2}-\frac{5}{4 \pi^{2}} x_{1}^{2}+\frac{5}{\pi} x_{1}-6\right)^{2}+10\left(1-\frac{1}{8 \pi}\right) \cos x_{1}+10
$$

$[-8.142,6.858]$

14. Goldstein-Price function

$f_{14}(\mathbf{x})=g(\mathbf{x}) \times h(\mathbf{x})$

$$
\begin{aligned}
g(\mathbf{x})= & 1+\left(x_{1}+x_{2}+1\right)^{2} \times & {[-2,2] \times } \\
& \left(19-14 x_{1}+3 x_{1}^{2}+6 x_{1} x_{2}+3 x_{2}^{2}\right) & {[-3,1] } \\
h(\mathbf{x})= & 30+\left(2 x_{1}-3 x_{2}\right)^{2} \times &
\end{aligned}
$$$$
\left(18-32 x_{1}+12 x_{1}^{2}+48 x_{2}-36 x_{1} x_{2}+27 x_{2}^{2}\right)
$$
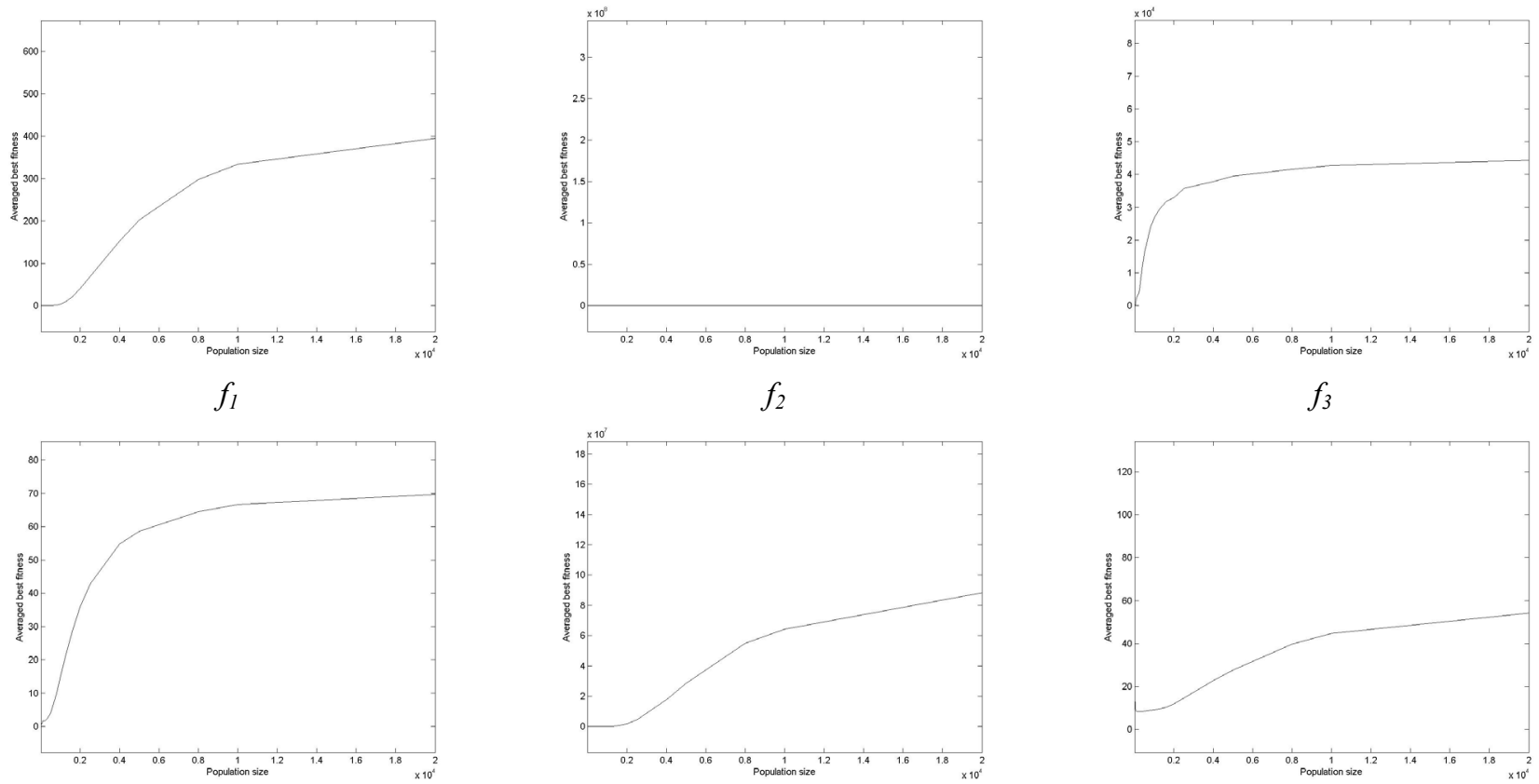

$f_{4}$

$f_{5}$

$f_{6}$
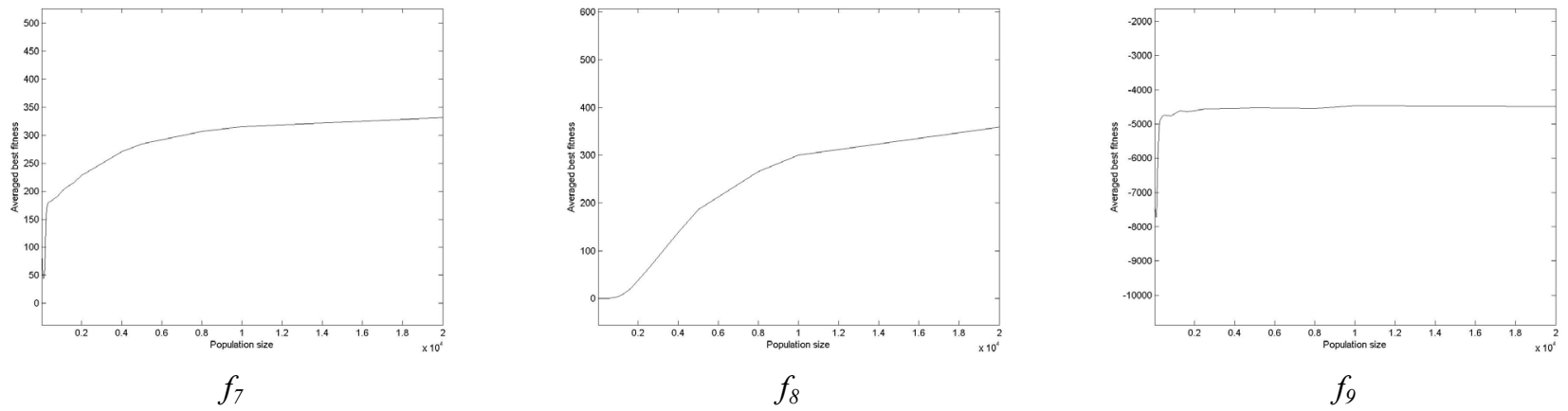


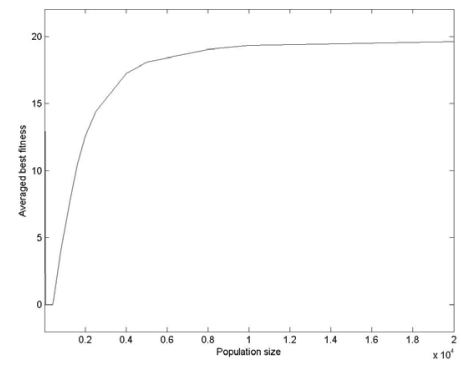

$f_{10}$

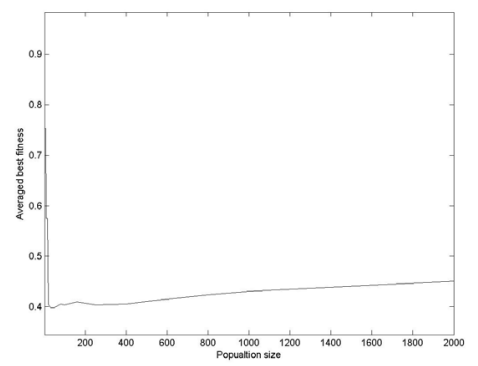

$f_{13}$

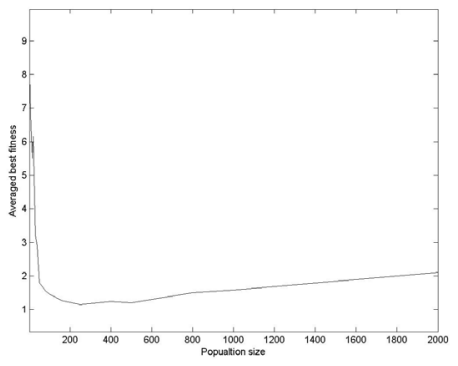

$f_{11}$

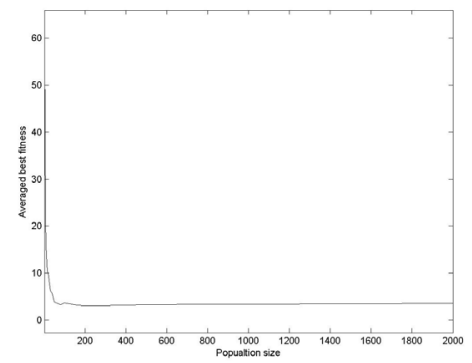

$f_{14}$

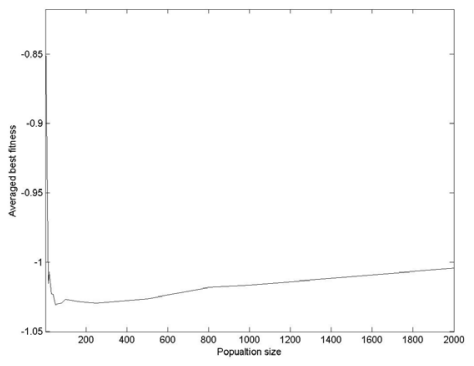

$f_{12}$

Figure 2. Averaged best fitness against population size for test functions $f_{1}-f_{14}$
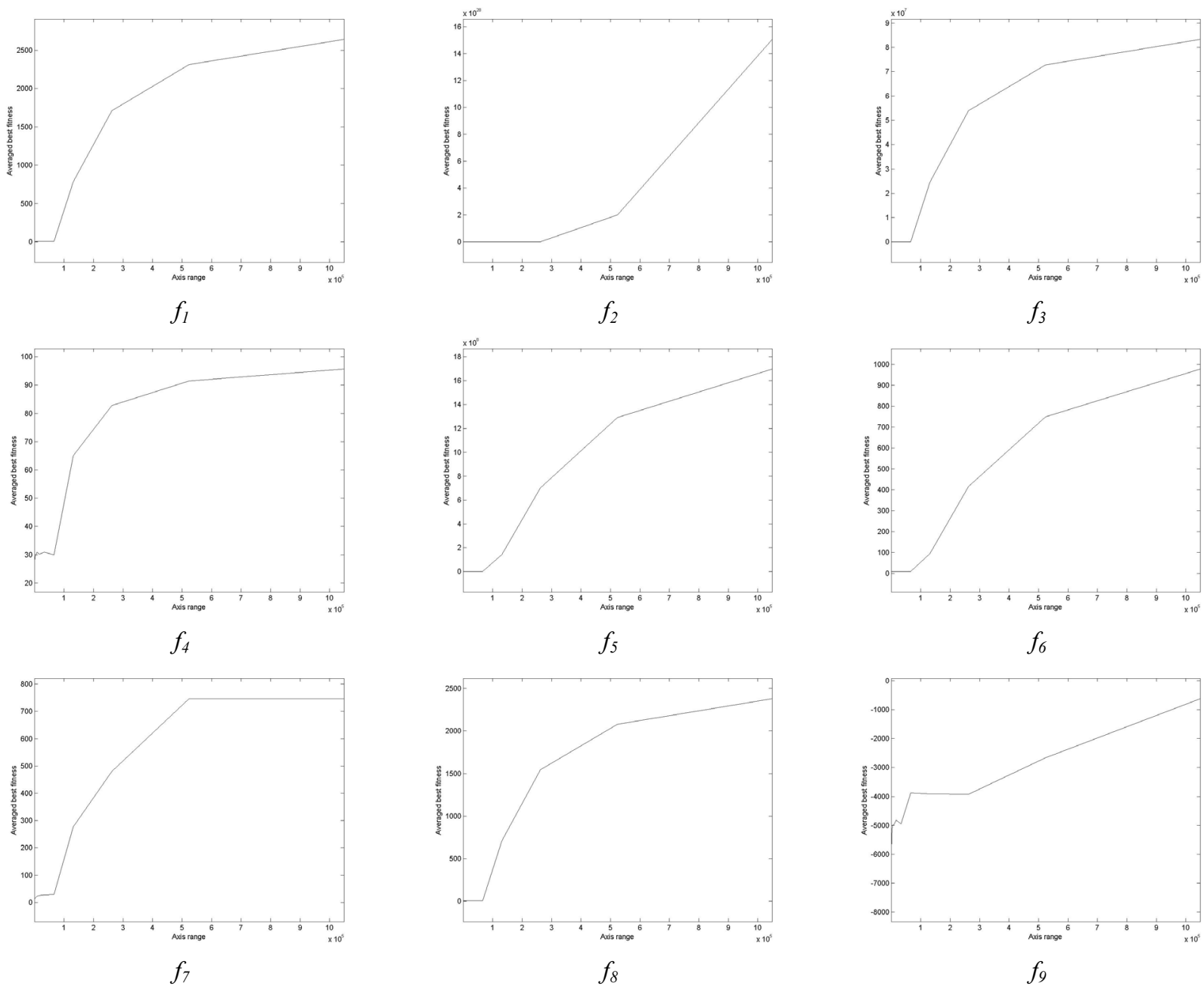

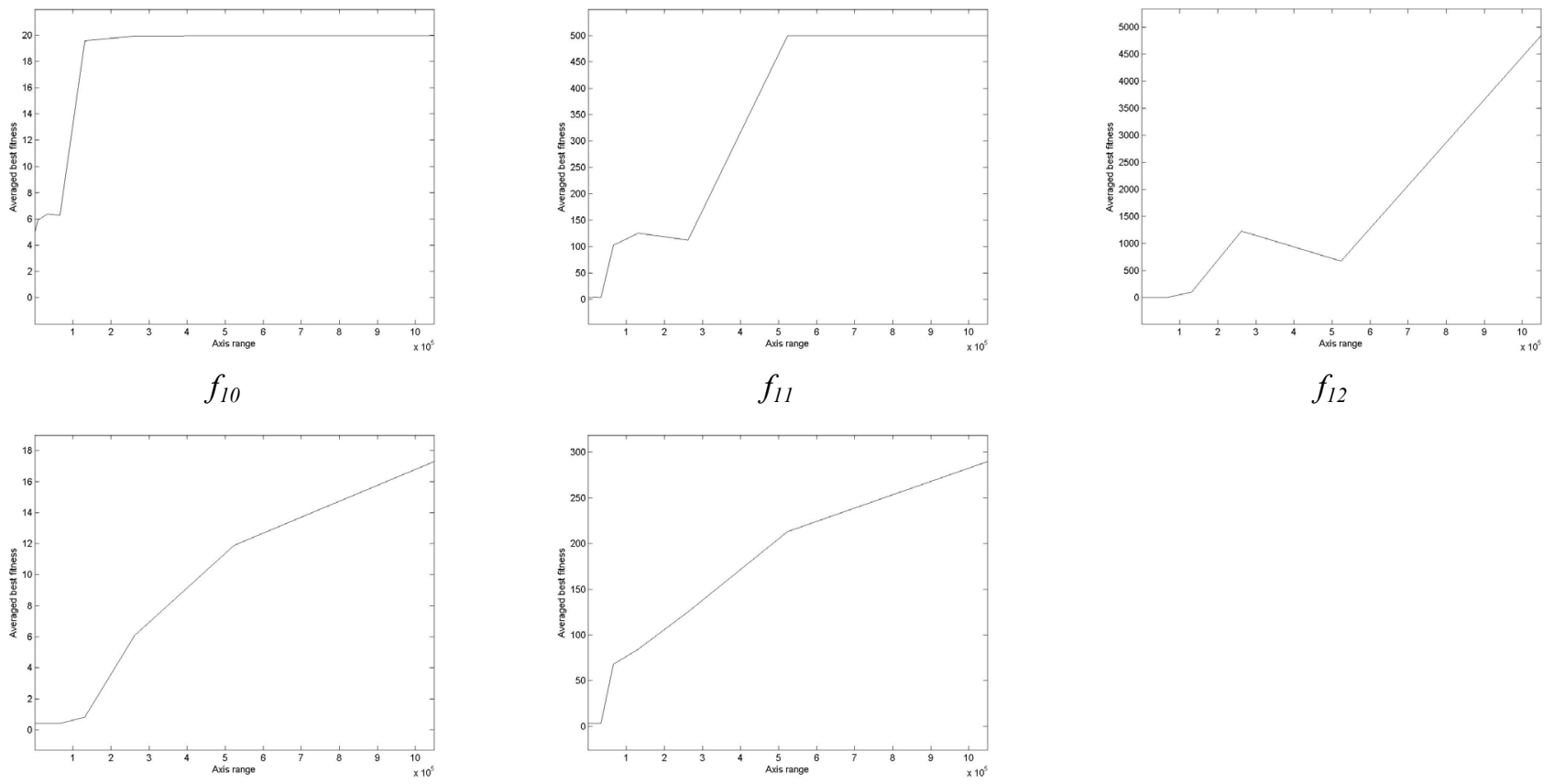

$f_{13}$

$f_{14}$

Figure 3. Averaged best fitness against axis resolution for test functions $f_{1}-f_{14}$

TABLE 6. AVERAGED BEST FITNESS AGAINST CROSSOVER RATE FOR TEST FUNCTIONS $f_{1}-f_{7}$

\begin{tabular}{|c|c|c|c|c|c|c|c|}
\hline$r_{x}$ & $f_{1}$ & $f_{2}$ & $f_{3}$ & $f_{4}$ & $f_{5}$ & $f_{6}$ & $f_{7}$ \\
\hline 0.00 & 177.81 & 61.08 & 24575.84 & 75.14 & 14732747.64 & 36.16 & 169.52 \\
\hline 0.05 & 0.00 & 0.75 & 8523.12 & 64.60 & 5937.73 & 15.36 & 61.39 \\
\hline 0.10 & 0.00 & 0.00 & 5986.56 & 58.56 & 816.94 & 11.55 & 39.55 \\
\hline 0.20 & 0.00 & 0.00 & 4121.60 & 48.62 & 711.10 & 9.38 & 24.14 \\
\hline 0.25 & 0.00 & 0.00 & 3699.92 & 43.64 & 578.92 & 9.05 & 21.23 \\
\hline 0.40 & 0.00 & 0.00 & 2777.20 & 36.62 & 648.40 & 8.75 & 17.61 \\
\hline 0.45 & 0.00 & 0.00 & 2671.68 & 35.76 & 634.44 & 8.67 & 16.61 \\
\hline 0.50 & 0.00 & 0.00 & 2528.44 & 35.96 & 583.29 & 8.67 & 16.41 \\
\hline
\end{tabular}

TABLE 7. AVERAGED BEST FITNESS AGAINST CROSSOVER RATE FOR TEST FUNCTIONS $f_{8}-f_{14}$

\begin{tabular}{|c|c|c|c|c|c|c|c|}
\hline$r_{x}$ & $f_{8}$ & $f_{9}$ & $f_{10}$ & $f_{11}$ & $f_{12}$ & $f_{13}$ & $f_{14}$ \\
\hline 0.00 & 158.77 & -8097.14 & 19.61 & 7.08 & -0.98 & 0.47 & 7.79 \\
\hline 0.05 & 0.09 & -11013.04 & 7.35 & 4.30 & -1.01 & 0.41 & 5.40 \\
\hline 0.10 & 0.00 & -12016.78 & 0.50 & 2.72 & -1.03 & 0.40 & 4.25 \\
\hline 0.20 & 0.00 & -12763.54 & 0.00 & 2.26 & -1.03 & 0.40 & 3.16 \\
\hline 0.25 & 0.00 & -12856.69 & 0.00 & 2.21 & -1.03 & 0.40 & 3.89 \\
\hline 0.40 & 0.00 & -13011.31 & 0.00 & 2.11 & -1.03 & 0.40 & 3.83 \\
\hline 0.45 & 0.00 & -13088.86 & 0.00 & 2.22 & -1.03 & 0.40 & 3.52 \\
\hline 0.50 & 0.00 & -12965.46 & 0.00 & 2.30 & -1.03 & 0.40 & 3.45 \\
\hline
\end{tabular}

